\title{
On the Unconditional Convergence of Wavelet Expansions for Continuous Functions
}

\author{
Naohiro Fukuda, Tamotu Kinoshita, and Toshio Suzuki
}

\begin{abstract}
In this paper, we study the unconditional convergence of wavelet expansions with Lipschitz wavelets. Especially with the Strömberg wavelet, we shall construct a counter example which shows that uniformly convergent wavelet expansions even for continuous functions do not always converge unconditionally in $L^{\infty}(\mathbf{R})$.
\end{abstract}

Keywords Unconditional Convergence, Wavelet Expansion, Strömberg Wavelet.

AMS Mathematics Subject Classification (2010) 42C15, 65T60

\section{Introduction}

Let $\Omega$ be an open set of $\mathbf{R}$. The space of bounded variations functions is denoted by $B V(\Omega)$ with the norm $\|f\|_{B V}:=\|f\|_{L^{1}}+V(f, \Omega)$, where $V$ is the total variation. The Sobolev space $W^{1,1}(\Omega)$ is a subspace of $B V(\Omega)$ and has the decay property at infinity for the unbounded domain $\Omega$ (see e.g., [10]). We remark that $B V(\Omega)$ allows jump-type discontinuities. When $\Omega$ is a bounded domain, the similar function spaces $B V(\Omega)$ and $W^{1,1}(\Omega)$ include the space of Lipschitz continuous functions $\operatorname{Lip}(\Omega)$, but not the space of Hölder continuous functions $C^{\alpha}(\Omega)$ with $0<\alpha<1$. In case of $\Omega=\mathbf{R}$, after modifying the function on a set of measure zero we find that $\operatorname{Lip}(\mathbf{R})=$ $W_{l o c}^{1, \infty}(\mathbf{R})$, and by the Sobolev embedding theorem the following set inclusions hold:

$$
\operatorname{Lip}(\mathbf{R}) \subset W_{l o c}^{1,1}(\mathbf{R}), \quad W^{1,1}(\mathbf{R}) \subset C^{0}(\mathbf{R}) \cap L^{\infty}(\mathbf{R}) .
$$

As for the Fourier expansion $f(t)=\sum_{j \in \mathbf{Z}} c_{j} e_{j}(t)$ on $\Omega$, the following results are well-known (see [9] etc.): 
$(i)_{F}$ If $f \in C^{\alpha}(\Omega)$ for $\alpha>1 / 2$, the Fourier series converges uniformly and absolutely, i.e., $\sum_{j \in \mathbf{Z}}\left|c_{j}\right|<\infty$.

$(\text { ii })_{F}$ If $f \in W^{1,1}(\Omega) \cap C^{\alpha}(\Omega)$ for $\alpha>0$, the Fourier series converges uniformly and absolutely, i.e., $\sum_{j \in \mathbf{Z}}\left|c_{j}\right|<\infty$. In fact, $W^{1,1}(\Omega)$ can be relaxed to $B V(\Omega)$.

$(\text { iii })_{F}$ For the function $f(t)=\sum_{n=1}^{\infty} \frac{\sin n t}{n \log (1+n)} \in W^{1,1}(\Omega)$ with $\Omega=(-\pi, \pi)$ its Fourier series does not converge absolutely.

For a Schauder basis $\left\{e_{j}(t)\right\}$, the ordering of the basis is important in the sense of stable convergence. For a Banach space $X, \sum_{j \in \mathbf{Z}} c_{j} e_{j}(t)$ converges to $f(t)$ unconditionally in $X$ if and only if $\sum_{\sigma(j) \in \mathbf{Z}} c_{j} e_{j}(t)$ converges to $f(t)$ in $X$ for all permutations $\sigma$ of $\mathbf{Z}$. In other words, for the sequence $\left\{\beta_{j}\right\} \subset\{1,-1\}$ the series $\sum_{j \in \mathbf{Z}} \beta_{j} c_{j} e_{j}(t)$ converges in $X$. Especially for a Hilbert space $X$ with an orthonormal basis $\left\{e_{j}(t)\right\}$, the unconditional convergence holds thanks to the Parseval's identity $\left\|\sum_{\sigma(j) \in \mathbf{Z}} c_{j} e_{j}\right\|_{X}^{2}=\sum_{\sigma(j) \in \mathbf{Z}}\left|c_{j}\right|^{2}$. If $X$ is a Banach space, the absolute convergence is stronger than the unconditional one. Noting that

$$
\left\|\sum_{j \in \mathbf{Z}}\left|c_{j}\right| e_{j}\right\|_{L^{\infty}(\Omega)}=\text { ess. } \sup _{t \in \Omega} \sum_{j \in \mathbf{Z}}\left|c_{j} e_{j}(t)\right|=\sum_{j \in \mathbf{Z}}\left|c_{j}\right|
$$

in case of the Banach space $X=L^{\infty}(\Omega)$ and $\left|e_{j}(t)\right| \equiv 1$, we find that the Fourier series converges to $f(t)$ unconditionally in $L^{\infty}(\Omega)$ under the assumptions in $(i)_{F}$ or $(i i)_{F}$. Here we pay attention to the fact that the Banach space $X=L^{\infty}(\Omega)$ for the convergence and the Banach space $\tilde{X}=C^{\alpha}(\Omega)$ or $W^{1,1}(\Omega)$ for the limit $f$ are different $(\tilde{X} \subset X)$ in the Fourier series.

Remark 1.1 We can take the Hilbert space $X=\tilde{X}=L^{2}(\Omega)$ for both the convergence and the limit, since the limit $f$ belongs to $L^{2}(\Omega)$ by the completeness of $L^{2}(\Omega)$. We need not assume any additional regularity for the limit $f$ unlike the case of $X=L^{\infty}(\Omega)$.

Remark 1.2 If $X=\tilde{X}=L^{p}(\Omega)$ except the Hilbert space $L^{2}(\mathbf{R})$, the Fourier series is not an unconditional basis (see [7], [9]).

Now we shall consider the wavelet expansion $f(t)=\sum_{j \in \mathbf{Z}} \sum_{k \in \mathbf{Z}} c_{j, k} \psi_{j, k}(t)$, where $\psi_{j, k}(t)=2^{j / 2} \psi\left(2^{j} t-k\right)$. We are concerned with the unconditionality of wavelet expansions except for the particular case of the Hilbert space $L^{2}(\mathbf{R})$. 
There are a lot of various wavelet bases $\psi$, although the Fourier basis consists of only the analytic function space $\mathcal{A}$. Therefore, we shall classify wavelets $\psi$ by a belonging function space (or set) $Y$ which restricts the regularity or the decay at infinity. Then the following results about wavelets are known:

$(i v)_{w}$ If $\psi \in Y=\left\{y \in C^{1}(\mathbf{R}) ;|y(t)|+\left|y^{\prime}(t)\right| \leq g(|t|)\right\}$ with a decreasing $g \in L^{1}[0, \infty)$ such that $|g(0)|<\infty$ and $\|\operatorname{tg}(\cdot)\|_{L^{1}[0, \infty)}<\infty,\left\{\psi_{j, k}(t)\right\}$ is an unconditional basis in $X=\tilde{X}=L^{p}(\mathbf{R})$ with $1<p<\infty$ (see [4]).

$(v)_{w}$ If $\psi \in Y=\{y \in \mathcal{A}(\mathbf{R}) ; \mathcal{F}[y]$ is characteristic functions of a finite sum of bounded closed intervals (unimodular wavelets) $\},\left\{\psi_{j, k}(t)\right\}$ is an unconditional basis in $X=\tilde{X}=L^{p}(\mathbf{R})$ with $1<p<\infty$ (see [1], [5]).

Let us choose the Banach space $X=\tilde{X}=W^{1,1}(\mathbf{R})$, and also $\psi \in Y=$ $W^{1,1}(\mathbf{R})$. The choices of $X=\tilde{X}=Y=W^{1,1}(\mathbf{R})$ give that

$$
\begin{aligned}
\sum_{j \in \mathbf{Z}} \sum_{k \in \mathbf{Z}}\left\|c_{j, k} \psi_{j, k}\right\|_{W^{1,1}} & \leq \sum_{j \in \mathbf{Z}} \sum_{k \in \mathbf{Z}} 2^{j / 2}\left|c_{j, k}\right|\left\|\psi\left(2^{j} \cdot-k\right)\right\|_{W^{1,1}} \\
& =\sum_{j \in \mathbf{Z}} \sum_{k \in \mathbf{Z}} 2^{j / 2}\left|c_{j, k}\right| \int_{\mathbf{R}}\left\{\left|\psi\left(2^{j} t-k\right)\right|+2^{j}\left|\psi^{\prime}\left(2^{j} t-k\right)\right|\right\} d t \\
& \leq\left(\sum_{j \in \mathbf{Z}} \sum_{k \in \mathbf{Z}} 2^{-j / 2}\left|c_{j, k}\right|\right)\|\psi\|_{L^{1}}+\left(\sum_{j \in \mathbf{Z}} \sum_{k \in \mathbf{Z}} 2^{j / 2}\left|c_{j, k}\right|\right)\left\|\psi^{\prime}\right\|_{L^{1}} \\
& \leq\left(\sum_{j \in \mathbf{Z}} \sum_{k \in \mathbf{Z}} 2^{|j| / 2}\left|c_{j, k}\right|\right)\|\psi\|_{W^{1,1}} .
\end{aligned}
$$

Thus, if the scalar series $\sum_{j \in \mathbf{Z}} \sum_{k \in \mathbf{Z}} 2^{|j| / 2}\left|c_{j, k}\right|$ converges, for $\psi \in W^{1,1}(\mathbf{R})$, the wavelet expansion $\sum_{j \in \mathbf{Z}} \sum_{k \in \mathbf{Z}} c_{j, k} \psi_{j, k}$ converges absolutely and also unconditionally in $W^{1,1}(\mathbf{R})$. Thus, we see the following basic observation:

Proposition 1.3 Assume that $\psi \in W^{1,1}(\mathbf{R})$. Then, the wavelet expansion $\sum_{j \in \mathbf{Z}} \sum_{k \in \mathbf{Z}} c_{j, k} \psi_{j, k}(t)$ converges to $f(t)$ unconditionally in $W^{1,1}(\mathbf{R})$ if the coefficients satisfy $\left\{2^{|j| / 2} c_{j, k}\right\}_{(j, k) \in \mathbf{Z}^{2}} \in \ell^{1}$.

Remark 1.4 Since the Sobolev embedding theorem gives $W^{1,1}(\mathbf{R}) \subset L^{2}(\mathbf{R})$, the coefficients $c_{j, k}:=\left(f, \psi_{j, k}\right)_{L^{2}}$ are well-defined.

It is not clear whether the condition $\left\{2^{|j| / 2} c_{j, k}\right\}_{(j, k) \in \mathbf{Z}^{2}} \in \ell^{1}$ really restricts the limit $f \in W^{1,1}(\mathbf{R})$. This does not conclude the unconditionality in 
$X=\tilde{X}=W^{1,1}(\mathbf{R})$, but indicates the possibility that there exists a counter example (non-unconditionality) if $f \notin W^{1,1}(\mathbf{R})$ and $\left\{2^{|j| / 2} c_{j, k}\right\}_{(j, k) \in \mathbf{Z}^{2}} \notin \ell^{1}$.

From (1), we see that the Sobolev space $W^{1,1}(\mathbf{R})$ can be regarded as a function space between $\operatorname{Lip}(\mathbf{R})$ (with a sufficient decay at infinity) and $C^{0}(\mathbf{R}) \cap L^{\infty}(\mathbf{R})$. For the non-unconditionality, our strategy is to find a suitable wavelet $\psi \in Y=\operatorname{Lip}(\mathbf{R})$ (with an exponential decay at infinity) and a particular function $f$ which belongs to $C^{0}(\mathbf{R}) \cap L^{\infty}(\mathbf{R})$ but not $W^{1,1}(\mathbf{R})$, i.e.,

$$
f \in \tilde{X}=\left\{C^{0}(\mathbf{R}) \cap L^{\infty}(\mathbf{R})\right\} \backslash W^{1,1}(\mathbf{R}),
$$

because the gap between the regularities of $\psi$ and $f$ possibly makes the unconditionality worse. Indeed, there is a gap between $\mathcal{A}$ and $C^{1 / 2}(\mathbf{R})$ for the basis and the limit of Fourier series. We shall take $X=L^{\infty}(\mathbf{R})$ as $\tilde{X}$ which is a little bit weak topology than $W^{1,1}(\mathbf{R})$. Thus, we prove the following result of the wavelet expansion which corresponding to $(i i i)_{F}$ in case of the Fourier expansion:

Theorem 1.5 There exists $f_{0} \in\left\{C^{0}(\mathbf{R}) \cap L^{\infty}(\mathbf{R})\right\} \backslash W^{1,1}(\mathbf{R})$ satisfying the following:

- $f_{0}$ has the wavelet expansion $f_{0}(t)=\sum_{j \in \mathbf{Z}} \sum_{k \in \mathbf{Z}} c_{j, k} \psi_{j, k}(t)$ in $L^{2}(\mathbf{R})$ for some $\psi \in \operatorname{Lip}(\mathbf{R})$ and $\left\{c_{j, k}\right\}_{(j, k) \in \mathbf{Z}^{2}} \in \ell^{2}$ such that $\left\{2^{|j| / 2} c_{j, k}\right\}_{(j, k) \in \mathbf{Z}^{2}} \notin$ $\ell^{1}$.

- $\sum_{j \in \mathbf{Z}} \sum_{k \in \mathbf{Z}} c_{j, k} \psi_{j, k}(t)$ converges to $f_{0}(t)$ uniformly and non-unconditionally in $L^{\infty}(\mathbf{R})$.

For the proof of Theorem 1.5, it is sufficient to construct a concrete $f_{0} \in\left\{C^{0}(\mathbf{R}) \cap L^{\infty}(\mathbf{R})\right\} \backslash W^{1,1}(\mathbf{R})$. The Strömberg wavelet $\psi^{S t} \in \operatorname{Lip}(\mathbf{R})$ has an exponential decay at infinity and enables us to construct $f_{0}$ simply (see Remark 2.2). Especially for the Franklin wavelet $\psi^{F r} \in \operatorname{Lip}(\mathbf{R})$, the following fact is known:

$(v i)_{w}\left\{\psi_{j, k}^{F r}(t)\right\}$ is an unconditional basis in $X=\tilde{X}=L^{p}(\mathbf{R})$ with $1<p<\infty$ (see Theorem 6.23 in $\S 5$ of [4]).

This holds for the spline wavelets of the same order. Therefore, we get the following also for $\psi^{S t} \in \operatorname{Lip}(\mathbf{R})$ : 
$(v i)_{w}^{\prime}\left\{\psi_{j, k}^{S t}(t)\right\}$ is an unconditional basis in $X=\tilde{X}=L^{p}(\mathbf{R})$ with $1<p<\infty$ (see Theorem 6.14 in $\S 5$ of [4]).

These two facts $(v i)_{w}$ and $(v i)_{w}^{\prime}$ are obtained with a small modification the $C^{1}$ assumption of $(i v)_{w}$. We remark that $\sum_{j \in \mathbf{Z}} \sum_{k \in \mathbf{Z}}\left|c_{j, k}\right| \psi_{j, k}^{S t}(t) \in X=L^{p}(\mathbf{R})$ with $1<p<\infty$ and $f_{0}$ is not a counter example for $(v i)_{w}^{\prime}$. The function space $X=L^{\infty}(\mathbf{R})$ in Theorem 1.5 is locally stronger than $X=L^{p}(\mathbf{R})$ with $1<p<\infty$ in $(v i)_{w}^{\prime}$. This causes the non-unconditionality even for the continuous function $f_{0}$.

\section{Proof of Theorem 1.5}

\subsection{Piecewise Linear Spline Wavelets}

The B-spline $N_{2}$ is defined by

$$
N_{2}(t)=\left\{\begin{array}{l}
t \text { for } 0 \leq t \leq 1 \\
2-t \text { for } 1 \leq t \leq 2 \\
0 \text { otherwise }
\end{array}\right.
$$

Piecewise linear spline wavelets can be given by

$$
\psi(t)=\sum_{k \in \mathbf{Z}} b_{k} N_{2}(2 t-k) .
$$

Then, we shall give the following function:

$$
f_{0}(t)=\sum_{j \in \mathbf{Z}} \sum_{k \in \mathbf{Z}} c_{j, k} \psi_{j, k}(t) \text { with } c_{j, k}=\left\{\begin{array}{c}
\frac{(-1)^{j}}{(j+1) 2^{\frac{j}{2}}} \text { for } j \geq 0 \text { and } k=0, \\
0 \text { otherwise. }
\end{array}\right.
$$

Our purpose is to prove $f_{0} \notin W^{1,1}(\mathbf{R})$ and the fact that $\sum_{j \in \mathbf{Z}} \sum_{k \in \mathbf{Z}} c_{j, k} \psi_{j, k}(t)$ converges to $f_{0}(t)$ uniformly and non-unconditionally in $L^{\infty}(\mathbf{R})$. Let us put $t_{n}=2^{-n}(n \geq 1)$. The function $f_{0}$ is rewritten as

$$
f_{0}(t)=\sum_{j=0}^{\infty} \frac{(-1)^{j}}{(j+1) 2^{\frac{j}{2}}} \psi_{j, 0}(t)=\sum_{j=0}^{\infty} \frac{(-1)^{j}}{j+1} \psi\left(2^{j} t\right)=\sum_{j=0}^{\infty} \sum_{k \in \mathbf{Z}} \frac{(-1)^{j}}{j+1} b_{k} N_{2}\left(2^{j+1} t-k\right) .
$$

Furthermore, using the fact that $\operatorname{supp} N_{2} \subset[0,2]$, we shall compute $f_{0}(t)$ for $t \in\left[2^{-n}, 2^{-n+1}\right]=\left[t_{n}, t_{n-1}\right]$ as follows: 
- Case $n=1)$ For $t \in\left[2^{-1}, 2^{0}\right]=\left[t_{1}, t_{0}\right]$ we have

$$
\begin{aligned}
f_{0}(t)= & \frac{(-1)^{0}}{0+1}\left\{b_{0} N_{2}\left(2^{0+1} t-0\right)+b_{1} N_{2}\left(2^{0+1} t-1\right)\right\} \\
& +\frac{(-1)^{1}}{1+1}\left\{b_{1} N_{2}\left(2^{1+1} t-1\right)+b_{2} N_{2}\left(2^{1+1} t-2\right)+b_{3} N_{2}\left(2^{1+1} t-3\right)\right\} \\
& +\frac{(-1)^{2}}{2+1}\left\{b_{3} N_{2}\left(2^{2+1} t-3\right)+b_{4} N_{2}\left(2^{2+1} t-4\right)+b_{5} N_{2}\left(2^{2+1} t-5\right)\right. \\
& \left.+\cdots b_{6} N_{2}\left(2^{2+1} t-6\right)+b_{7} N_{2}\left(2^{2+1} t-7\right)\right\} \\
= & \sum_{j=0}^{\infty} \sum_{k=2^{j}-1}^{2^{j+1}-1} \frac{(-1)^{j}}{j+1} b_{k} N_{2}\left(2^{j+1} t-k\right),
\end{aligned}
$$

here we used that $2^{0+1} t \in[1,2], 2^{1+1} t \in[2,4], 2^{2+1} t \in[4,8]$, and $2^{j+1} t \in$ $\left[2^{j}, 2^{j+1}\right]$.

- Case $n=2)$ For $t \in\left[2^{-2}, 2^{-1}\right]=\left[t_{2}, t_{1}\right]$ we have

$$
\begin{aligned}
f_{0}(t)= & \frac{(-1)^{0}}{0+1}\left\{b_{-1} N_{2}\left(2^{0+1} t+1\right)+b_{0} N_{2}\left(2^{0+1} t-0\right)\right\} \\
& +\frac{(-1)^{1}}{1+1}\left\{b_{0} N_{2}\left(2^{1+1} t-0\right)+b_{1} N_{2}\left(2^{1+1} t-1\right)\right\} \\
& +\frac{(-1)^{2}}{2+1}\left\{b_{1} N_{2}\left(2^{2+1} t-1\right)+b_{2} N_{2}\left(2^{2+1} t-2\right)+b_{3} N_{2}\left(2^{2+1} t-3\right)\right\} \\
& +\cdots \\
= & \frac{(-1)^{0}}{0+1}\left\{b_{-1} N_{2}\left(2^{0+1} t+1\right)+b_{0} N_{2}\left(2^{0+1} t-0\right)\right\} \\
& +\sum_{j=1}^{\infty} \sum_{k=2^{j-1}-1}^{2^{j}-1} \frac{(-1)^{j}}{j+1} b_{k} N_{2}\left(2^{j+1} t-k\right),
\end{aligned}
$$

here we used that $2^{0+1} t \in\left[2^{-1}, 1\right], 2^{1+1} t \in[1,2], 2^{2+1} t \in[2,4]$, and $2^{j+1} t \in$ $\left[2^{j-1}, 2^{j}\right]$.

- Case $n=3)$ For $t \in\left[2^{-3}, 2^{-2}\right]=\left[t_{3}, t_{2}\right]$ we have

$$
f_{0}(t)=\frac{(-1)^{0}}{0+1}\left\{b_{-1} N_{2}\left(2^{0+1} t+1\right)+b_{0} N_{2}\left(2^{0+1} t-0\right)\right\}
$$




$$
\begin{aligned}
& +\frac{(-1)^{1}}{1+1}\left\{b_{-1} N_{2}\left(2^{1+1} t+1\right)+b_{0} N_{2}\left(2^{1+1} t-0\right)\right\} \\
& +\frac{(-1)^{2}}{2+1}\left\{b_{0} N_{2}\left(2^{2+1} t-0\right)+b_{1} N_{2}\left(2^{2+1} t-1\right)\right\} \\
& +\cdots \\
& =\sum_{j=0}^{1} \frac{(-1)^{j}}{j+1}\left\{b_{-1} N_{2}\left(2^{j+1} t+1\right)+b_{0} N_{2}\left(2^{j+1} t-0\right)\right\} \\
& +\sum_{j=2}^{\infty} \sum_{k=2^{j-2}-1}^{2^{j-1}-1} \frac{(-1)^{j}}{j+1} b_{k} N_{2}\left(2^{j+1} t-k\right),
\end{aligned}
$$

here we used that $2^{0+1} t \in\left[2^{-2}, 2^{-1}\right], 2^{1+1} t \in\left[2^{-1}, 1\right], 2^{2+1} t \in[1,2]$, and $2^{j+1} t \in\left[2^{j-2}, 2^{j-1}\right]$.

Thus, if $n \geq 2$, for $t \in\left[2^{-n}, 2^{-n+1}\right]=\left[t_{n}, t_{n-1}\right]$ we recursively have

$$
\begin{aligned}
f_{0}(t)= & \sum_{j=0}^{n-2} \frac{(-1)^{j}}{j+1}\left\{b_{-1} N_{2}\left(2^{j+1} t+1\right)+b_{0} N_{2}\left(2^{j+1} t-0\right)\right\} \\
& +\sum_{j=n-1}^{\infty} \sum_{k=2^{j-n+1}-1}^{2^{j-n+2}-1} \frac{(-1)^{j}}{j+1} b_{k} N_{2}\left(2^{j+1} t-k\right) .
\end{aligned}
$$

Since $2^{j+1} t \in\left[2^{j-n+1}, 2^{j-n+2}\right] \subset[0,1]$ for $0 \leq j \leq n-2$, we see that

$$
N_{2}\left(2^{j+1} t+1\right)=2-\left(2^{j+1} t+1\right)=1-2^{j+1} t, \quad N_{2}\left(2^{j+1} t-0\right)=2^{j+1} t
$$

and get

$$
\begin{aligned}
f_{0}(t)= & \sum_{j=0}^{n-2} \frac{(-1)^{j}}{j+1}\left\{\left(1-2^{j+1} t\right) b_{-1}+2^{j+1} t b_{0}\right\} \\
& +\sum_{j=n-1}^{\infty} \sum_{k=2^{j-n+1}-1}^{2^{j-n+2}-1} \frac{(-1)^{j}}{j+1} b_{k} N_{2}\left(2^{j+1} t-k\right) .
\end{aligned}
$$

Remark 2.1 In the same way we also get for $t \in\left[-2^{-n+1},-2^{-n}\right]=\left[-t_{n-1},-t_{n}\right]$

$$
\begin{aligned}
f_{0}(t)= & \sum_{j=0}^{n-2} \frac{(-1)^{j}}{j+1}\left\{\left(1-2^{j+1} t\right) b_{-1}-2^{j+1} t b_{-2}\right\} \\
& +\sum_{j=n-1}^{\infty} \sum_{k=-2^{j-n+2}-1}^{-2^{j-n+1}-1} \frac{(-1)^{j}}{j+1} b_{k} N_{2}\left(2^{j+1} t-k\right) .
\end{aligned}
$$




\subsection{Unbounded Variation}

It is sufficient to show that $f_{0} \notin B V(\mathbf{R})$ instead of $f_{0} \notin W^{1,1}(\mathbf{R})$, since $W^{1,1}(\mathbf{R}) \subset B V(\mathbf{R})$. Especially when $t=t_{n}$, noting that $N_{2}\left(2^{j+1} t_{n}-k\right)=$ $\delta_{1,2^{j+1} t_{n}-k}$, that is, the summation with respect to $k$ runs over only $k=$ $2^{j+1} t_{n}-1=2^{j-n+1}-1$, (3) can be changed into

$$
f_{0}\left(t_{n}\right)=\sum_{j=0}^{n-2} \frac{(-1)^{j}}{j+1}\left\{\left(1-2^{j-n+1}\right) b_{-1}+2^{j-n+1} b_{0}\right\}+\sum_{j=n-1}^{\infty} \frac{(-1)^{j}}{j+1} b_{2^{j-n+1}-1} .
$$

Hence it follows that for $n \geq 3$

$$
\begin{aligned}
f_{0}\left(t_{n-1}\right)-f_{0}\left(t_{n}\right)= & \sum_{j=0}^{n-3} \frac{(-1)^{j}}{j+1}\left\{\left(1-2^{j-n+2}\right) b_{-1}+2^{j-n+2} b_{0}\right\}+\sum_{j=n-2}^{\infty} \frac{(-1)^{j}}{j+1} b_{2^{j-n+2}-1} \\
& -\sum_{j=0}^{n-2} \frac{(-1)^{j}}{j+1}\left\{\left(1-2^{j-n+1}\right) b_{-1}+2^{j-n+1} b_{0}\right\}-\sum_{j=n-1}^{\infty} \frac{(-1)^{j}}{j+1} b_{2^{j-n+1}-1} \\
= & \sum_{j=0}^{n-2} \frac{(-1)^{j}}{j+1}\left\{\left(1-2^{j-n+2}\right) b_{-1}+2^{j-n+2} b_{0}\right\}-\frac{(-1)^{n-2}}{n-1} b_{0} \\
& +\sum_{j=n-1}^{\infty} \frac{(-1)^{j}}{j+1} b_{2^{j-n+2}-1}+\frac{(-1)^{n-2}}{n-1} b_{0} \\
& -\sum_{j=0}^{n-2} \frac{(-1)^{j}}{j+1}\left\{\left(1-2^{j-n+1}\right) b_{-1}+2^{j-n+1} b_{0}\right\}-\sum_{j=n-1}^{\infty} \frac{(-1)^{j}}{j+1} b_{2^{j-n+1}-1} \\
= & \sum_{j=0}^{n-2} \frac{(-1)^{j}}{j+1} 2^{j-n+1}\left(b_{0}-b_{-1}\right)+\sum_{j=n-1}^{\infty} \frac{(-1)^{j}}{j+1}\left(b_{2^{j-n+2}-1}-b_{2^{j-n+1}-1}\right) .
\end{aligned}
$$

Our next task is to find a suitable wavelet whose coefficients $b_{k}$ satisfy $\sum_{n=1}^{\infty}\left|f_{0}\left(t_{n-1}\right)-f_{0}\left(t_{n}\right)\right|=\infty$.

In particular, for convenience, we shall choose the Strömberg wavelet $\psi^{S t} \in \operatorname{Lip}(\mathbf{R})$ given by

$$
\psi^{S t}(t)=\sum_{k \in \mathbf{Z}} b_{k} N_{2}(2 t-k),
$$

where

$$
b_{k}=\left\{\begin{array}{l}
-4(\sqrt{3}-2)^{k} \quad \text { if } k \geq 1, \\
-\frac{5}{2}+\frac{\sqrt{3}}{2} \text { if } k=0 \\
-(2-\sqrt{3})^{-\frac{k}{2}}\left(\cos \frac{k \pi}{2}+\sqrt{2} \sin \frac{k \pi}{2}\right) \quad \text { if } k \leq-1,
\end{array}\right.
$$


(see [3]). Noting that

$$
\sum_{j=0}^{n-2} \frac{(-2)^{j}}{j+1}=2^{-1} \int_{0}^{2} \frac{1-(-y)^{n-1}}{1+y} d y=2^{-1} \log 3-2^{n-1} \int_{0}^{1} \frac{(-z)^{n-1}}{1+2 z} d z
$$

we can rewrite

$$
\begin{aligned}
f_{0}\left(t_{n-1}\right)-f_{0}\left(t_{n}\right)= & \left(b_{0}-b_{-1}\right) 2^{1-n} \sum_{j=0}^{n-2} \frac{(-2)^{j}}{j+1}+\frac{(-1)^{n-1}}{n}\left(b_{1}-b_{0}\right) \\
& +\sum_{j=n}^{\infty} \frac{(-1)^{j}}{j+1}\left(b_{2^{j-n+2}-1}-b_{2^{j-n+1}-1}\right) \\
= & \left(b_{-1}-b_{0}\right)\left(\int_{0}^{1} \frac{(-z)^{n-1}}{1+2 z} d z-2^{-n} \log 3\right)+\frac{(-1)^{n-1}}{n}\left(b_{1}-b_{0}\right) \\
& +\sum_{j=n}^{\infty} \frac{(-1)^{j}}{j+1}\left(b_{2^{j-n+2}-1}-b_{2^{j-n+1}-1}\right) .
\end{aligned}
$$

Especially, for $n=2 m+1(m \geq 1)$ we obtain

$$
\begin{aligned}
t_{2 m}-t_{2 m+1}= & 2^{-2 m-1}, \\
f_{0}\left(t_{2 m}\right)-f_{0}\left(t_{2 m+1}\right)= & \left(b_{-1}-b_{0}\right) \int_{0}^{1} \frac{z^{2 m}}{1+2 z} d z+\frac{b_{1}-b_{0}}{2 m+1} \\
& -\left(b_{-1}-b_{0}\right) 2^{-2 m-1} \log 3+\sum_{j=2 m+1}^{\infty} \frac{(-1)^{j}}{j+1}\left\{b_{2^{j-2 m+1}-1}-b_{2^{j-2 m}-1}\right\} \\
=: & I+I I-I I I+I V .
\end{aligned}
$$

Using $b_{-1}-b_{0}=\frac{1}{2}(3+\sqrt{3})$ and $b_{1}-b_{0}=\frac{3}{2}(7-3 \sqrt{3})$, we get

$$
\begin{aligned}
I+I I & \geq\left(b_{-1}-b_{0}\right) \int_{0}^{1} \frac{z^{2 m}}{1+2} d z+\frac{b_{1}-b_{0}}{2 m+1}>\frac{13(2-\sqrt{3})}{6(m+1)} \\
|I V| & \leq \frac{1}{2 m+2} \sum_{j=2 m+1}^{\infty}\left|b_{2^{j-2 m+1}-1}-b_{2^{j-2 m}-1}\right| \\
& =\frac{2}{(m+1)(2-\sqrt{3})} \sum_{j=2 m+1}^{\infty}\left\{(2-\sqrt{3})^{2^{j-2 m}}-(2-\sqrt{3})^{2^{j-2 m+1}}\right\} \\
& \leq \frac{2}{(m+1)(2-\sqrt{3})}(2-\sqrt{3})^{2^{(2 m+1)-2 m}}=\frac{2(2-\sqrt{3})}{m+1} .
\end{aligned}
$$


Since $|I I I|<\frac{2-\sqrt{3}}{7(m+1)}$ for $m \geq 2$, there exists $c>0$ such that for $m \geq 2$

$$
\begin{aligned}
\left|f_{0}\left(t_{2 m}\right)-f_{0}\left(t_{2 m+1}\right)\right| & \geq|I+I I+I V|-|I I I| \geq|I+I I-| I V||-|I I I| \\
& \geq \frac{c}{m+1} .
\end{aligned}
$$

Since $\sum_{n=1}^{\infty}\left|f_{0}\left(t_{n-1}\right)-f_{0}\left(t_{n}\right)\right| \geq \sum_{m=1}^{\infty}\left|f_{0}\left(t_{2 m}\right)-f_{0}\left(t_{2 m+1}\right)\right|=\infty$, we find that $f_{0} \notin B V(\mathbf{R})$. Therefore we can conclude that $f_{0} \notin W^{1,1}(\mathbf{R})$.

Remark 2.2 Thanks to the Strömberg wavelet $\psi^{S t} \in \operatorname{Lip}(\mathbf{R})$, we can know that $|I+I I+I V| \neq 0$. The information of the exact values of $b_{k}$ is required to find (5). Therefore, it would be difficult to get (5) for general piecewise linear spline wavelets $\psi \in \operatorname{Lip}(\mathbf{R})$ or even for the Franklin wavelet $\psi^{\text {Fr }} \in \operatorname{Lip}(\mathbf{R})$ whose values of $b_{k}$ are very complicated (see [3]).

\subsection{Continuity and Uniform Convergence}

As for the continuity (at $t=0$ ), with

$$
f_{0}(0)=\sum_{j=0}^{\infty} \sum_{k \in \mathbf{Z}} \frac{(-1)^{j}}{j+1} b_{k} N_{2}\left(2^{j+1} 0-k\right)=\sum_{j=0}^{\infty} \frac{(-1)^{j}}{j+1} b_{-1},
$$

by (3) we get

$$
\begin{aligned}
\left|f_{0}(t)-f_{0}(0)\right| \leq & \left|\sum_{j=n-1}^{\infty} \frac{(-1)^{j}}{j+1}\right|\left|b_{-1}\right|+\left|\sum_{j=0}^{n-2} \frac{(-1)^{j}}{j+1} 2^{j+1}\right|\left|b_{0}-b_{-1}\right| t \\
& +\left|\sum_{j=n-1}^{\infty} \sum_{k=2^{j-n+1}-1}^{2^{j-n+2}-1} \frac{(-1)^{j}}{j+1} b_{k} N_{2}\left(2^{j+1} t-k\right)\right| \\
=: & I^{\prime}+I I^{\prime}+I I I^{\prime} .
\end{aligned}
$$

We can estimate $I^{\prime}, I I^{\prime}$ and $I I I^{\prime}$ as follows:

$$
\begin{aligned}
I^{\prime} & =\left|\sum_{j=n-1}^{\infty} \frac{(-1)^{j}}{j+1}\right|(\sqrt{3}-1)=\left|\int_{0}^{1} \frac{(-z)^{n-1}}{1+z} d z\right|(\sqrt{3}-1) \\
& \leq \int_{0}^{1} \frac{z^{n-1}}{1+0} d z(\sqrt{3}-1) \leq \frac{C}{n}
\end{aligned}
$$




$$
\begin{aligned}
I I^{\prime} & \leq\left|\sum_{j=0}^{n-2} \frac{(-2)^{j}}{j+1}\right| \cdot 2\left|b_{0}-b_{-1}\right| t_{n-1} \\
& =\left|2^{-1} \log 3-2^{n-1} \int_{0}^{1} \frac{(-z)^{n-1}}{1+2 z} d z\right| \cdot 2\left|-\frac{1}{2}(3+\sqrt{3})\right| 2^{-n+1} \\
& \leq\left(2^{-1} \log 3+2^{n-1} \int_{0}^{1} \frac{z^{n-1}}{1+2 \cdot 0} d z\right) \cdot(3+\sqrt{3}) 2^{-n+1} \leq \frac{C}{n}, \\
I I I^{\prime} & \left.\leq \sum_{j=n-1}^{\infty} \sum_{k=2^{j-n+1}-1}^{2^{j-n+2}-1} \frac{\left|b_{k}\right|}{j+1}\left|b_{k}\right|\right) \sum_{k=2^{j-n+1}-1}^{2^{j-n+2}-1} 1 \\
& \leq \frac{1}{n} \sum_{j=n-1}^{\infty}\left(\sup _{2^{j-n+1}-1 \leq k \leq 2^{j-n+2}-1} C\right. \\
& \leq \frac{1}{n} \sum_{j=n-1}^{\infty} 4(2-\sqrt{3})^{2^{j-n+1}-1}\left(2^{j-n+1}+1\right) \leq \frac{C}{n} .
\end{aligned}
$$

Thus we find that $\left|f_{0}(t)-f_{0}(0)\right| \rightarrow 0$ as $n \rightarrow \infty$ for $t \in\left[2^{-n}, 2^{-n+1}\right]=$ $\left[t_{n}, t_{n-1}\right]$. This means the right continuity of $f_{0}$. Similarly, the left continuity of $f_{0}$ follows from (4) instead of (3), and we get $f_{0} \in C^{0}(\mathbf{R})$.

Remark 2.3 We remark that $f_{0}$ is not only continuous but also uniformly continuous. More precisely, $f_{0}$ satisfies $\left|f_{0}(t)-f_{0}(s)\right| \leq C / \log |t-s|^{-1}$ for $0<|t-s|<1 / 2$, that is log-Hölder continuous.

Remark 2.4 In general, $f_{0}$ is continuous if $f_{J} \rightarrow f_{0}$ uniformly, but the converse does not hold. It is known that $f_{J} \rightarrow f_{0}$ uniformly if $\left\{f_{J}(t)\right\}_{J}$ is uniformly equicontinuous and $f_{J}(t) \rightarrow f_{0}(t)$ pointwise. Let us take the sequence of partial sums

$$
f_{J}(t)=\sum_{j=0}^{J} \frac{(-1)^{j}}{(j+1) 2^{\frac{j}{2}}} \psi_{j, 0}^{S t}(t) .
$$

Similarly, we also find that $\left|f_{J}(t)-f_{J}(0)\right| \rightarrow 0$ as $n \rightarrow \infty$ for $t \in\left[t_{n}, t_{n-1}\right]$. We remark that the corresponding $I_{J}^{\prime}, I I_{J}^{\prime}$ and $I I I_{J}^{\prime}$ tend to 0 independently of $J$. This means that $\left\{f_{J}(t)\right\}_{J}$ is uniformly equicontinuous. We see that $f_{J}\left(t_{0}\right) \rightarrow f_{0}\left(t_{0}\right)$ for a fixed $t_{0} \neq 0$, since $\left|b_{k}\right|$ is rapidly decreasing at $\pm \infty$ and $f_{J}\left(t_{0}\right)=\sum_{j=0}^{J} \frac{(-1)^{j}}{(j+1) 2^{\frac{j}{2}}} \psi_{j, 0}^{S t}\left(t_{0}\right)=\sum_{j=0}^{J} \sum_{k \in \mathbf{Z}} \frac{(-1)^{j}}{j+1} b_{k} N_{2}\left(2^{j+1} t_{0}-k\right) \sim \sum_{j=0}^{J} \frac{(-1)^{j}}{j+1} b_{\left[2^{j+1} t_{0}\right]}$, 
where $[\alpha]$ is the largest integer not greater than $\alpha$. Meanwhile, we immediately see that $f_{J}(0) \rightarrow f_{0}(0)$ as an alternating series. Thus, $f_{J}(t) \rightarrow f_{0}(t)$ pointwise and we can conclude that $f_{J} \rightarrow f_{0}$ uniformly.

\subsection{Non-unconditional Convergence}

The non-unconditional convergence implies that there exists a sequence $\beta_{j} \in$ $\{1,-1\}$ such that the series $\sum_{j \in \mathbf{Z}} \beta_{j} c_{j} e_{j}(t)$ does not converge. In order to know the non-unconditional convergence of $f_{0}(t)=\sum_{j=0}^{\infty} \frac{(-1)^{j}}{(j+1) 2^{\frac{j}{2}}} \psi_{j, 0}^{S t}(t)$, with $\beta_{j}=\overline{c_{j}} /\left|c_{j}\right|$ especially for $\sum_{j \in \mathbf{Z}} \beta_{j} c_{j} e_{j}(t)$ we shall consider the divergence of

$$
\tilde{f}_{0}(t):=\sum_{j=0}^{\infty}\left|\frac{(-1)^{j}}{(j+1) 2^{\frac{j}{2}}}\right| \psi_{j, 0}^{S t}(t) .
$$

We remark that we can not deal with $\sum_{j=0}^{\infty}\left|\frac{(-1)^{j}}{(j+1)^{\frac{j}{2}}}\right|\left|\psi_{j, 0}^{S t}(t)\right|$ instead of $\tilde{f}_{0}(t)$. Let us define the interval $I_{J}=\left(2^{-J-3}, 2^{-J-3}+2^{-J-4}\right)$ for $J \geq 1$. Taking $L^{\infty}(\mathbf{R})$-norm, we have

$$
\begin{aligned}
\left\|\tilde{f}_{0}\right\|_{L^{\infty}(\mathbf{R})} & \geq\left\|\tilde{f}_{0}\right\|_{L^{\infty}\left(I_{J}\right)}=\left\|\sum_{j=0}^{\infty} \frac{\psi_{j, 0}^{S t}(t)}{(j+1) 2^{\frac{j}{2}}}\right\|_{L^{\infty}\left(I_{J}\right)} \\
& \geq\left\|\sum_{j=0}^{J-1} \frac{\psi_{j, 0}^{S t}(t)}{(j+1) 2^{\frac{j}{2}}}\right\|_{L^{\infty}\left(I_{J}\right)}-\left\|\sum_{j=J}^{\infty} \frac{\psi_{j, 0}^{S t}(t)}{(j+1) 2^{\frac{j}{2}}}\right\|_{L^{\infty}\left(I_{J}\right)} \\
& =: L_{J}-M_{J} .
\end{aligned}
$$

We note that $0<2^{j+1} t-k<2$ if $N_{2}\left(2^{j+1} t-k\right) \neq 0$, i.e., $2^{j+1} t-2<k<2^{j+1} t$. Therefore we may consider $\left[2^{j+1} t\right]-1 \leq k \leq\left[2^{j+1} t\right]$. As for the 1 st term, we get

$$
\begin{aligned}
L_{J} & \geq \underset{t \in I_{J}}{\operatorname{ess.sup}} \sum_{j=0}^{J-1} \frac{\psi_{j, 0}^{S t}(t)}{(j+1) 2^{\frac{j}{2}}}=\underset{t \in I_{J}}{\operatorname{ess} . \sup _{j=0}} \sum_{j-1}^{J-1} \frac{1}{j+1} \sum_{k \in \mathbf{Z}} b_{k} N_{2}\left(2^{j+1} t-k\right) \\
& =\underset{t \in I_{J}}{\operatorname{ess.sup}} \sum_{j=0}^{J-1} \frac{1}{j+1}\left\{b_{-1} N_{2}\left(2^{j+1} t+1\right)+b_{0} N_{2}\left(2^{j+1} t-0\right)\right\} \\
& =\underset{t \in I_{J}}{\operatorname{ess.sup}} \sum_{j=0}^{J-1} \frac{1}{j+1}\left\{b_{-1}\left(1-2^{j+1} t\right)+b_{0} 2^{j+1} t\right\}
\end{aligned}
$$




$$
=\underset{t \in I_{J}}{\operatorname{ess} . \sup _{j=0}} \sum_{j-1}^{J-1} \frac{1}{j+1}\left\{b_{-1}-2^{j+1} t\left(b_{-1}-b_{0}\right)\right\},
$$

here we used that $0<2^{j+1} t \leq \frac{3}{8}$ for $0 \leq j \leq J$ and $t \in I_{J}$. Since $b_{-1}=$ $\sqrt{4-2 \sqrt{3}}=\sqrt{3}-1$ and $b_{-1}-b_{0}=\frac{\sqrt{3}+3}{2}$, we see that

$$
\begin{aligned}
L_{J} & \geq \sum_{j=0}^{J-1} \frac{1}{j+1}\left\{b_{-1}-2^{j+1} \cdot\left(2^{-J-3}+2^{-J-4}\right) \cdot\left(b_{-1}-b_{0}\right)\right\} \\
& \geq \sum_{j=0}^{J-1} \frac{1}{j+1}\left\{b_{-1}-2^{(J-1)+1} \cdot\left(2^{-J-3}+2^{-J-4}\right) \cdot\left(b_{-1}-b_{0}\right)\right\} \\
& \geq \sum_{j=0}^{J-1} \frac{c}{j+1}(c>0) .
\end{aligned}
$$

As for the 2nd term, noting that $\left|b_{k}\right|$ is decreasing for $k \geq 0$, we get

$$
\begin{aligned}
& M_{J} \leq \underset{t \in I_{J}}{\operatorname{ess.sup}} \sum_{j=J}^{\infty} \frac{\left|\psi_{j, 0}^{S t}(t)\right|}{(j+1) 2^{\frac{j}{2}}}=\underset{t \in I_{J}}{\operatorname{ess} . \sup } \sum_{j=J}^{\infty} \frac{1}{j+1}\left|\sum_{k \in \mathbf{Z}} b_{k} N_{2}\left(2^{j+1} t-k\right)\right| \\
& =\underset{t \in I_{J}}{\operatorname{ess} . \sup } \sum_{j=J}^{\infty} \frac{1}{j+1} \mid b_{\left[2^{j+1} t\right]-1} N_{2}\left(2^{j+1} t-\left[2^{j+1} t\right]+1\right) \\
& +b_{\left[2^{j+1} t\right]} N_{2}\left(2^{j+1} t-\left[2^{j+1} t\right]\right) \\
& \leq \underset{t \in I_{J}}{\operatorname{ess} . \sup } \sum_{j=J}^{\infty} \frac{\left|b_{\left[2^{j+1} t\right]-1}\right|+\left|b_{\left[2^{j+1} t\right]}\right|}{j+1} \\
& \leq \frac{C}{J}+\underset{t \in I_{J}}{\operatorname{ess} . \sup _{j=J+2}} \sum_{j=}^{\infty} \frac{2\left|b_{\left[2^{j+1} t\right]-1}\right|}{j+1} \leq \frac{C}{J}+\sum_{j=J+2}^{\infty} \frac{2\left|b_{2^{j-J-2}-1}\right|}{j+1}
\end{aligned}
$$

here we used that $\left[2^{j+1} t\right]-1 \geq\left[2^{j-J-2}\right]-1=2^{j-J-2}-1(\geq 0)$ for $j \geq J+2$ and $t \in I_{J}$. Moreover, we easily see that

$$
M_{J} \leq \frac{C}{J}+c_{1}+\sum_{h=2}^{\infty} \frac{8(2-\sqrt{3})^{2^{h}-1}}{J+h+2} \leq C^{\prime}+\sum_{h=2}^{\infty} \frac{8(2-\sqrt{3})^{h}}{1+1+2} \leq C^{\prime \prime},
$$

where $C^{\prime}$ is independent of $J$. Thus, it follows that

$$
\left\|\tilde{f}_{0}\right\|_{L^{\infty}(\mathbf{R})} \geq L_{J}-M_{J} \geq \sum_{j=0}^{J-1} \frac{c}{j+1}-C^{\prime \prime} .
$$

This holds for all $J \geq 0$, that is, $\left\|\tilde{f}_{0}\right\|_{L^{\infty}(\mathbf{R})}=\infty$. 
Remark 2.5 If we take only $B^{0}(\mathbf{R})$-norm (sup-norm) instead of $L^{\infty}(\mathbf{R})$ norm (ess.sup-norm), by substituting $t=0$ we immediately find that

$\left\|\tilde{f}_{0}\right\|_{B^{0}(\mathbf{R})} \geq\left|\sum_{j=0}^{\infty}\right| \frac{(-1)^{j}}{(j+1) 2^{\frac{j}{2}}}\left|\psi_{j, 0}^{S t}(0)\right|=\left|\sum_{j=0}^{\infty} \frac{\sum_{k \in \mathbf{Z}} b_{k} N_{2}(-k)}{j+1}\right|=\sum_{j=0}^{\infty} \frac{b_{-1}}{j+1}=\infty$.

In the above estimate of $\left\|\tilde{f}_{0}\right\|_{L^{\infty}(\mathbf{R})}$, we used the sequence of the interval $\left\{I_{J}\right\}$ since the essential supremum excludes the measure zero set $\{t=0\}$. We also remark that $\sum_{j=0}^{\infty}\left|\frac{(-1)^{j}}{(j+1) 2^{\frac{j}{2}}}\right| \psi_{j, 0}^{S t}(t)$ does not converge to $\tilde{f}_{0}(t)$ uniformly (while $\sum_{j=0}^{\infty} \frac{(-1)^{j}}{(j+1) 2^{\frac{j}{2}}} \psi_{j, 0}^{\text {St }}(t)$ converges to $f_{0}(t)$ uniformly $)$.

\section{Concluding Remarks}

- We have considered a continuous wavelet with sufficient decay. In general, such a wavelet is difficult to be exactly represented in the time domain. We paid attention to a little-known fact that the Strömberg wavelet has simple exact values at nodes. The sequence of those simple exact values decays rapidly and plays an important role to derive some estimates in the proof. So, we have utilized the Strömberg wavelet effectively in this paper.

- In our construction, the unbounded variation of a function causes the nonunconditional convergence of a wavelet expansion. We remark that the proof would become simpler if one considered a discontinuous function with the unbounded variation. But we can not expect the uniform convergence, if discontinuous functions are expanded by continuous wavelets. So, we have succeeded to construct a continuous function whose wavelet expansion converges uniformly and non-unconditionally in $L^{\infty}$.

\section{References}

[1] R. Ashino and T. Mandai, Wavelet bases for microlocal filtering and the sampling theorem in $L_{p}\left(\mathbf{R}^{n}\right)$, Appl. Anal., 82, No. 1, 1-24 (2003).

[2] I. Daubechies, Ten lectures on wavelets, CBMS-NSF Regional Conference Series in Applied Mathematics, 61, SIAM, Philadelphia, PA, 1992. 
[3] N. Fukuda and T. Kinoshita, On non-symmetric orthogonal spline wavelets, Southeast Asian Bull. Math., 36, No. 3, 319-341 (2012).

[4] E. Hernández and G. Weiss, A first course on wavelets, CRC Press, Boca Raton, FL, 1996.

[5] T. Hoshiro, Unconditional convergence of wavelet expansions (Japanese) (Kyoto, 2004). Sūrikaisekikenkyūsho Kōkyūroku, No. 1385, 94-104 (2004).

[6] Y. Meyer, Wavelets and operators. Translated from the 1990 French original by D. H. Salinger. Cambridge Studies in Advanced Mathematics, 37, Cambridge University Press, Cambridge, 1992.

[7] R. E. A. C. Paley and A. Zygmund, On some series of functions, Proc. of the Cambridge Phil. Soc., 34, 337-357, 458-474 (1930) and 28, 190-205 (1932).

[8] J. O. Strömberg, Ondelettes à localisation exponentielles, Proc. Conference in Harmonic Analysis in honor of Antoni Zygmund, 475-493 (1983).

[9] A. Zygmund, Trigonometric series, Cambridge University Press, 1959.

[10] W. P. A. Ziemer, Weakly differentiable functions. Sobolev spaces and functions of bounded variation. Graduate Texts in Mathematics, 120. Springer-Verlag, New York, 1989., Cambridge University Press, 1959.

Addresses of the authors

Naohiro Fukuda, National Institute of Technology, Matsue College, Matsue Shimane, 690-8518, Japan

e-mail: fukuda@matsue-ct.jp

Tamotu Kinoshita, Institute of Mathematics, Tsukuba University, Tsukuba Ibaraki 305-8571, Japan

e-mail: kinosita@math.tsukuba.ac.jp

Toshio Suzuki, Institute of Mathematics, Tsukuba University, Tsukuba Ibaraki 305-8571, Japan

e-mail: toshio-s@math.tsukuba.ac.jp 